\title{
NODAL NONCOMMUTATIVE JORDAN ALGEBRAS
}

BY

\author{
ROBERT H. OEHMKE
}

1. A finite-dimensional power-associative algebra $\mathfrak{A}$ is said to be nodal [6] if every element of $\mathfrak{A}$ can be written as $\alpha 1+z$ where $\alpha \in \mathfrak{F}, 1$ is the unity element of $\mathfrak{A}$ and $z$ is nilpotent and if the set of all nilpotent elements is not a subalgebra of $\mathfrak{A}$.

In $[3 ; 4]$, Kokoris has shown that every simple nodal noncommutative Jordan algebra of characteristic $p \neq 2$ has the form $\mathfrak{A}=\mathfrak{F} 1+\mathfrak{N}$ with $\mathfrak{N}^{+}=\mathfrak{F}\left[x_{1}, \cdots, x_{n}\right]$ for some $n$ where the generators are all nilpotent of index $p$ and the multiplication is associative. If $f$ and $g$ are two elements of $\mathfrak{A}$ then the multiplication table of $\mathfrak{U}$ is given by

$$
f g=f \circ g+\frac{1}{2} \sum_{i, j} \frac{\partial f}{\partial x_{i}} \circ \frac{\partial g}{\partial x_{j}} \circ c_{i j}
$$

where the circle product is the product in $\mathfrak{A}^{+}$and

$$
c_{i j}=x_{i} x_{j}-x_{j} x_{i}
$$

In [7] Schafer considers nodal noncommutative Jordan algebras defined by a skew-symmetric bilinear form (i.e., $c_{i j} \in \mathfrak{F}$ ) and those with two generators. All of these algebras are Lie-admissible (i.e., $\mathfrak{A}^{-}$is a Lie algebra). Schafer obtained the derivation algebras of these algebras defined by a skew-symmetric bilinear form.

Here, we examine all simple nodal noncommutative Jordan algebras that are Lie-admissible over a field $\mathfrak{F}$ of characteristic $p \neq 2$. First a set of generators is obtained having properties suitable for further study. This set of generators is then used to find the algebras $D(\mathfrak{U})$ of derivatives of $\mathfrak{A}$ and the algebras adj $\mathfrak{U}^{-}$and $\left(\operatorname{adj} \mathfrak{U}^{-}\right)^{\prime}$. Schafer has shown that all of the simple Lie algebras defined by Block [1] can be realized as (adj $\left.\mathfrak{U}^{-}\right)^{\prime}$ for some $\mathfrak{A}$ that is simple, nodal noncommutative Jordan and Lie-admissible. Hence we have obtained a somewhat different formulation of these algebras. The question remains whether all of these algebras, $\left(\operatorname{adj} \mathfrak{A}^{-}\right)^{\prime}$, are in the class defined by Block. It is our intention to investigate this question in a subsequent paper.

2. We define the mapping $D_{y}=D(y)$ by

$$
x D_{y}=x y-y x .
$$

Received by the editors April 19, 1963. 
Then $D_{y}=R_{y}-L_{y}$ where $R_{y}$ and $L_{y}$ are the right and left multiplications by $y$ on $\mathfrak{A}$.

A derivation of an algebra $\mathfrak{B}$ is a linear transformation $T$ on $\mathfrak{B}$ into $\mathfrak{B}$ such that for $x, y \in \mathfrak{B}$

$$
(x y) T=(x T) y+x(y T) .
$$

Since $\mathfrak{A}^{-}$is a Lie algebra $D_{y}$ is the right multiplication by $y$ of $\mathfrak{A}^{-}$and is a derivation of $\mathfrak{A}^{-}$. By expanding

$$
2(x \circ y) D_{z}-2\left(x D_{z}\right) \circ y-2 x \circ\left(y D_{z}\right)
$$

in terms of the multiplication of $\mathfrak{A}$ and using the flexible law

$$
(x y) z+(z y) x=x(y z)+z(y x)
$$

we see that $D_{z}$ is also a derivation of $\mathfrak{A}^{+}$and hence of $\mathfrak{A}$.

It is well known [2, p. 108] that any set of $n$ elements of $\mathfrak{R}$ whose cosets form a basis of the $n$-dimensional space $\mathfrak{N}-\mathfrak{N} \circ \mathfrak{N}$ can serve as a set of generators of $\mathfrak{A}^{+}$. This result shall be our chief tool in the proof of the following theorem.

THEOREM 1. Let $\mathfrak{A}$ be a simple, Lie-admissible, nodal noncommutative Jordan algebra over a base field $\mathfrak{F}$ of characteristic $p \neq 2$. If $\mathfrak{A}^{+}$has an even number of generators then a set of generators $x_{1}, \cdots, x_{2 r}$ can be chosen for $\mathfrak{A}^{+}$so that

$$
\begin{aligned}
x_{i} D\left(x_{i+r}\right) & =1+\alpha_{i} x_{i}^{p-1} \circ x_{i+r}^{p-1}, & & i=1, \cdots, r, \\
x_{i} D\left(x_{j}\right) & =0, & & j \neq i+r,
\end{aligned}
$$

with $\alpha_{i} \in \mathfrak{F}$. If $\mathfrak{A}^{+}$has an odd number of generators then a set of generators $x_{1}, \cdots, x_{2 r+1}$ can be chosen for $\mathfrak{H}^{+}$so that (1) is satisfied and

$$
\begin{aligned}
x_{2 r+1} D\left(x_{j}\right) & =0, \quad j=1, \cdots, 2 r-2, \\
x_{2 r+1} D\left(x_{2 r}\right) & =x_{2 r-1}^{p-1} \circ\left(1+\beta x_{2 r+1}^{p-1}\right), \\
x_{2 r+1} D\left(x_{2 r-1}\right) & =x_{2 r}^{p-1} \circ \alpha\left(1+\beta x_{2 r+1}^{p-1}\right),
\end{aligned}
$$

with $\alpha$ and $\beta$ in $\mathfrak{F}$.

Proof. Since $\mathfrak{A}$ is simple $\mathfrak{N}$ can not be an ideal of $\mathfrak{A}^{-}$. For if $\mathfrak{N}$ is an ideal of $\mathfrak{U}^{-}$then since it is an ideal of $\mathfrak{A}^{+}$it would be closed under both the operations $R_{y}-L_{y}$ and $R_{y}+L_{y}$ for $y \in \mathfrak{A}$. Therefore it would be also an ideal of $\mathfrak{A}$. Hence there must be a pair of generators $x$ and $y$ such that $y D_{x}$ is nonsingular. Since $y$ can be replaced by $\alpha y$ for any $\alpha$ in $\mathfrak{F}$ we assume

$$
y D_{x}=1+m \circ y^{k}=b^{-1} .
$$

We also assume $y$ has been chosen so that $k$ is a maximum. If $k<p-1$ then letting $q=(y-1 /(k+1)) y^{k+1} \circ m \circ b$ we have 


$$
\begin{aligned}
q D_{x} & =1-\frac{1}{k+1} y^{k+1} \circ(m \circ b) D_{x} \\
& =1+q^{k+1} \circ m^{\prime}
\end{aligned}
$$

which contradicts the choice of $k$. Hence we can assume in (3) that $k=p-1$.

We now write (3) as

$$
y D_{x}=1+y^{p-1} \circ x^{t} \circ m^{\prime}=b^{-1}
$$

and assume that $y$ and $x$ have been chosen so that $t$ is a maximum. If $t<p-1$ then, as above, we can replace $x$ by $x-1 /(t+1) \circ x^{t+1} \circ m^{\prime} \circ y^{p-1} \circ b$ to obtain a contradiction to our choice of $t$. Hence we can assume $x$ and $y$ have been chosen so that

$$
y D_{x}=1+m_{y} \circ y^{p-1} \circ x^{p-1} .
$$

If $z$ is a third generator, in the same way that we altered the generator $y$, we can add an element $q$ of $y \circ \mathfrak{A}$ to $z$ to obtain the property

$$
(z+q) D_{x} \in y^{p-1} \circ \mathfrak{A} \text {. }
$$

Hence we assume that all generators $z$ different from $x$ and $y$ have been chosen so that

$$
z D_{x}=y^{p-1} \circ m_{z} .
$$

Since for any $q$ in $\mathfrak{U}$ we have $D_{q}$ a derivation of both $\mathfrak{U}$ and $\mathfrak{U}^{-}$then

$$
z D_{y} D_{x}=z D_{x} D_{y}-y D_{x} D_{z}
$$

If (4) and (5) are substituted in (6) we have

$$
\begin{aligned}
z D_{y} D_{x}= & y^{p-1} \circ m_{z} D_{y}+y^{p-2} \circ x^{p-1} \circ m_{y} \circ y D_{z} \\
& -y^{p-1} \circ x^{p-1} \circ m_{y} D_{z} .
\end{aligned}
$$

But the right-hand side of (7) is in $y^{p-2} \circ \mathfrak{A}$; so also is the left-hand side. From (4) and (5) the only possible way for this to happen is to have

$$
z D_{y}=n_{0}+y^{p-1} \circ n_{1}
$$

in which $n_{i}$ is independent of $y$.(i.e., $n_{i}$ is a polynomial in which $y$ does not appear.) In (7) this implies

$$
\begin{gathered}
n_{0} D_{x}-y^{p-2} \circ n_{1}=y^{p-1} \circ m_{z} D_{y}-y^{p-2} \circ x^{p-1} \circ m_{y} \circ n_{0} \\
-y^{p-1} \circ x^{p-1} \circ m_{y} D_{z}
\end{gathered}
$$

and

$$
n_{1}=x^{p-1} \circ m_{y} \circ n_{0}
$$


Write $n_{0}=x^{k} \circ t$. If $k<p-1$ we can replace the generator $z$ by the generator $z+1 /(k+1) \circ x^{k+1} \circ t=z^{\prime}$ to get

$$
\begin{aligned}
z^{\prime} D_{y} & =n_{0}+y^{p-1} \circ m_{y} \circ n_{0} \circ x^{p-1}+x^{k} \circ t \circ x D_{y}+\frac{1}{k+1} x^{k+1} \circ t D_{y} \\
& =y^{p-1} \circ x^{p-1} \circ m_{y} \circ n_{0}+\frac{1}{k+1} x^{k+1} \circ t D_{y} \\
& =n_{0}^{\prime}+y^{p-1} \circ x^{k+1} \circ m_{y} \circ n_{1}^{\prime}
\end{aligned}
$$

in which $n_{i}^{\prime}$ is again independent of $y$. Note that if (5) holds and $z$ is replaced by a generator $z+q$ in which $q$ is independent of $y$ then (5) will be retained.

Again arguing on the maximum value of $k$ that can be obtained in the expression $n_{0}=x^{k} \circ t$ we can conclude that $k=p-1, n_{1}=0$ and

$$
\begin{aligned}
& z D_{y}=x^{p-1} \circ n_{z} \\
& z D_{x}=y^{p-1} \circ m_{z}
\end{aligned}
$$

in which $n_{z}$ is independent of $y$.

Identity (7) can now be reduced to

$$
x^{p-1} \circ n_{z} D_{x}=y^{p-1} \circ m_{z} D_{y}-y^{p-1} \circ x^{p-1} \circ m_{y} D_{z} \text {. }
$$

For a particular choice of a set of generators including $x$ and $y$ satisfying (4) assume there are two distinct generators $w$ and $z$ (both satisfying (9)). Write

$$
m_{z}=\Sigma x^{i} \circ m_{i}, \quad m_{0}=\Sigma w^{i} \circ n_{i} \text {. }
$$

(When obvious, we shall omit index and range of the summation.) Then

$$
m_{z} D_{y}=-\sum i x^{i-1} \circ m_{i}+\sum x^{i} \circ m_{i} D_{y}-m_{0} \circ y^{p-1} \circ x^{p-1} .
$$

But from (10) $y^{p-1} \circ m_{z} D_{y} \in x \circ \mathfrak{A}$. Therefore

$$
\begin{array}{r}
-y^{p-1} \circ m_{1}+y^{p-1} \circ m_{0} D_{y} \in x \circ \mathfrak{A}, \\
-y^{p-1} \circ m_{1}+y^{p-1} \circ \sum i w^{i-1} \circ n_{i} \circ w D_{y}+\sum w^{i} \circ n_{i} D_{y} \in x \circ \mathfrak{A} .
\end{array}
$$

If $w$ is replaced as a generator by $w^{\prime}=w-x$ then (9) still holds for $z$ and hence so do the corresponding relationships (12). Note that if $P(w)$ is a polynomial in $w$ then $P(w)-P(w+x) \in x \circ \mathfrak{A}$ and $w D_{y}-(w+x) D_{y}-1 \in x \circ \mathfrak{A}$. If we write $q^{\prime}$ for $q=q(w)$ with $w$ replaced by $w+x$ then $w^{\prime} D_{x}=y^{p-1} \circ m_{z}^{\prime} ; m_{z}^{\prime}=\sum x^{i} \circ m_{i}^{\prime} ;$ $m_{0}^{\prime}=\sum w^{i} \circ n_{i}^{\prime}$ and from (11) we have

$$
\begin{aligned}
0 & \equiv-y^{p-1} \circ m_{1}^{\prime}+y^{p-1} \circ \sum i w^{i-1} \circ n_{i}^{\prime} \circ w^{\prime} D_{y}+\sum w^{i} \circ n_{i}^{\prime} D_{y} \\
& \equiv-y^{p-1} \circ m_{1}+y^{p-1} \circ \sum i w^{i-1} \circ n_{i} \circ w^{\prime} D_{y}+\sum w^{i} \circ n_{i} D_{y} \\
& \equiv-y^{p-1} \circ m_{1}+y^{p-1} \circ \sum i w^{i-1} \circ n_{i} \circ\left(w D_{y}-1\right)+\sum w^{i} \circ n_{i} D_{y}
\end{aligned}
$$

modulo $x \circ \mathfrak{A}$. 
But this implies $y^{p-1} \circ \sum_{i w^{1-1}} \circ n_{i} \in x \circ \mathfrak{A}$. Therefore $y^{p-1} \circ n_{i} \in x \circ \mathfrak{A}$ for $i>0$.

Now assume that in (11) we have chosen the $m_{i}$ to be independent of $x$. Then since $m_{z}$ is independent of $y$ and $m_{0}$ is independent of $x$ we have $n_{i}=0$ for $i>0$. Hence $m_{0}$ is independent of $w$. Since $w$ was arbitrary we must have $m_{0}$ a polynomial in the single generator $z$. But then $y^{p-1} \circ m_{0} D_{y} \in x \circ \mathfrak{A}$ by (9) and $y^{p-1} \circ m_{1} \in x \circ \mathfrak{A}$ by (12). However $m_{1}$ is independent of $x$ and $y$. Hence $m_{1}=0$.

Once again looking at (12) we have

$$
y^{p-1} \circ m_{z} D_{y} \equiv-y^{p-1} \circ \sum i x^{i-1} \circ m_{i}+y^{p-1} \circ \sum x^{i} \circ m_{i} D_{y} \equiv 0
$$

modulo $x^{p-1} \circ \mathfrak{A}$. With $m_{0} D_{y}$ in $x^{p-1} \circ \mathfrak{A}$ and $m_{1}=0$ we see that $m_{2}=\cdots$ $=m_{p-1}=0$ and $m_{z}=m_{0}$ is a polynomial in $z$ with coefficients in $\mathfrak{F}$. Similarly we obtain $n_{z}$ as a polynomial in $z$ with coefficients in $\mathfrak{F}$. Therefore if the number of generators is greater than or equal to 4 and they have been picked so that (4) and (9) hold then $m_{z}$ and $n_{z}$ in (9) are polynomials in the single generator $z$.

However if $z$ and $w$ are two generators distinct from $x$ and $y$ then $z$ can be replaced as a generator by $z+w$. Indentity (9) still holds, i.e.,

$$
\begin{aligned}
& (z+w) D_{x}=y^{p-1} \circ m_{z+w}, \\
& (z+w) D_{y}=x^{p-1} \circ n_{z+w}
\end{aligned}
$$

in which $m_{z+w}$ and $n_{z+w}$ are polynomials in the single generator $(z+w)$. But $m_{z+w}=m_{z}+m_{w}$ and $n_{z+w}=n_{z}+n_{w}$. For these sums to be polynomials in $(z+w), m_{z}$ and $n_{z}$ must be of degree at most 1 . If $z$ is replaced by $z+z^{2}$ then (9) still holds for the generator $\left(z+z^{2}\right)$. In particular $m_{z}+2 z \circ m_{z}$ is of degree at most 1 in $\left(z+z^{2}\right)$. Write $m_{z}$ as $\alpha+\beta z$ and $m_{z}+2 m_{z} \circ m_{z}$ as $\gamma+\delta\left(z+z^{2}\right)$. Then $\beta=2 \alpha$. Since $z$ was arbitrary we must also have $\delta=2 \gamma$. But the same relationships that gave us $\beta=2 \alpha$ also give us $\delta=4 \gamma$, i.e., $\delta=\gamma=\alpha=\beta=0$. Hence $m_{z}=0$ and in the same manner $n_{z}=0$.

We still assume we have at least two generators $z$ and $w$ distinct from $x$ and $y$. We also assume that they have been chosen so that

$$
z D_{x}=z D_{y}=w D_{x}=w D_{y}=0 .
$$

We must have

$$
w D_{z} D_{x}=w D_{x} D_{z}-z D_{x} D_{w}
$$

and therefore $\left(w D_{z}\right) D_{x}=0$. This implies that $w D_{z}$ is independent of $y$. Similarly $\left(w D_{z}\right) D_{y}=0$ and $w D_{z}$ is independent of $x$. Then if we assume that all the generators distinct from $x$ and $y$ have been chosen so that their product in $\mathfrak{A}^{-}$by either $x$ or $y$ is 0 , we can assume that the polynomials over $\mathfrak{F}$ in these generators is an Ideal $\mathfrak{I}$ of $\mathfrak{U}^{-}$. But then $\mathfrak{I} \circ \mathfrak{U}$ is an ideal in both $\mathfrak{U}^{-}$and $\mathfrak{U}^{+}$and hence in $\mathfrak{A}$. Therefore $\mathfrak{I}$ must contain a nonsingular element. This means that there are two generators $w$ and $z$, distinct from $x$ and $y$, such that $w D_{z}$ is nonsingular. 
At this point we reconsider the polynomial $m_{y}$ obtained in (4). If the generators $x, y, z, w$ have been chosen so that (4) and (13) hold and $z$ and $w$ are such that $w D_{z}$ is nonsingular then $(7)$ reduces to

$$
y^{p-1} \circ x^{p-1} \circ m_{y} D_{z}=0 .
$$

Therefore $m_{y} D_{z}$ is 0 since it is independent of both $x$ and $y$. But this implies that $m_{y}$ is independent of $w$ and by symmetry $m_{y}$ is independent of $z$. If $t$ is a fifth generator then either $t D_{z}$ or $(w+t) D_{z}$ is nonsingular. In either case we see that $m_{y}$ is also independent of $t$. Hence $m_{y} \in \mathfrak{F}$.

We can now proceed in $\mathfrak{I}$ (defined above) with the same argument as above to obtain the result of the theorem for the even-dimensional case.

In the odd dimensional case we can proceed with the above argument until we are presented with an $\mathfrak{I}$ which is the set of polynomials over $\mathfrak{F}$ in three generators, say $x, y$ and $z$. Again by the previous arguments we can assume that $x, y$ and $z$ have been chosen so that

$$
\begin{aligned}
& y D_{x}=1+y^{p-1} \circ x^{p-1} \circ m_{y}, \\
& z D_{x}=y^{p-1} \circ m_{z}, \\
& z D_{y}=x^{p-1} \circ n_{z} .
\end{aligned}
$$

Consider (7). We have

$$
x^{p-1} \circ n_{z} D_{x}=y^{p-1} \circ m_{z} D_{y}-y^{p-1} \circ x^{p-1} \circ m_{y} D_{z} .
$$

Since $m_{y}$ is a polynomial in $x, y$ and $z$ then $y^{p-1} \circ x^{p-1} \circ m_{y} D_{z}=0$. Also since $m_{z}$ is independent of $y$ and by (9) $m_{z} D_{y}$ is independent of $y$ we must have $m_{z} D_{y} \in x^{p-1} \circ \mathfrak{A}$. This implies that $m_{z}$ is independent of $x$. Hence $y^{p-1} \circ m_{z} D_{y}$ $=\partial m_{z} / \partial z \circ x^{p-1} \circ y^{p-1}$ and $x^{p-1} \circ m_{z} D_{x}=\partial n_{z} / \partial z \circ x^{p-1} \circ y^{p-1}$. From (14) we have

$$
\frac{\partial n_{z}}{\partial z}=\frac{\partial m_{z}}{\partial z}
$$

If both $n_{z}$ and $m_{z}$ are singular then $z D_{x}$ and $z D_{y}$ are in $z \circ \mathfrak{A}$. Hence $z \circ \mathfrak{A}$ is an ideal of $\mathfrak{U}^{-}$and $\mathfrak{U}^{+}$. Since this denies the simplicity of $\mathfrak{A}$ we must have either $m_{z}$ or $n_{z}$ nonsingular. Assume $m_{z}=1+q$ in which $q \in \mathfrak{N}$. Then if $l$ is a polynomial in $z$ over $\mathfrak{F}$ we have

$$
(z+l) D_{x}=y^{p-1} \circ(1+q)+y^{p-1} \circ \frac{\partial l}{\partial z} \circ(1+q) .
$$

Clearly, $l$ can be chosen so that $\partial l / \partial z \circ(1+q) \equiv w$ modulo $z^{p-1} \circ \mathfrak{A}$. Hence we can assume

$$
z D_{x}=y^{p-1}+\beta y^{p-1} \circ z^{p-1}
$$


in which $\beta \in \mathfrak{F}$. Now since $m_{z}$ is nonsingular the solutions of (15) are of the form $n_{z}=\alpha m_{z}$. Hence

$$
z D_{y}=\alpha x^{p-1} \circ\left(1+\beta z^{p-1}\right) \text {. }
$$

Again, let $l$ be a polynomial in $z$ over $\mathfrak{F}$. Then

$$
\left(y+x^{p-1} \circ l\right) D_{x}=1+y^{p-1} \circ x^{p-1} \circ\left[m_{y}+\frac{\partial l}{\partial z} \circ\left(1+\beta z^{p-1}\right)\right] \text {. }
$$

Write $m_{y}=\gamma+z \circ t$ and $l=\delta z+z^{2} \circ l^{\prime}$ in which

$$
\frac{\partial\left(z^{2} \circ l^{\prime}\right)}{\partial z}+z \circ t
$$

is a multiple of $z^{p-1}$. Then

$$
\frac{\partial\left(z^{2} \circ l^{\prime}\right)}{\partial z} \circ\left(1+\beta z^{p-1}\right)+z \circ t
$$

is also a multiple of $z^{p-1}$. Now choose $\delta$ so that

$$
\delta\left(1+\beta_{z}^{p-1}\right)+\frac{\partial\left(z^{2} \circ l^{\prime}\right)}{\partial z} \circ\left(1+\beta z^{p-1}\right)+z \circ t
$$

is a constant. We now have

$$
\left(y+x^{p-1} \circ l\right) D_{x}=1+\gamma \circ x^{p-1} \circ y^{p-1} .
$$

Since $\left(y+x^{p-1} \circ l\right)^{p-1} \circ x^{p-1}=y^{p-1} \circ x^{p-1}$ we can assume the generator $y$ can be chosen so that

$$
y D_{x}=1+\gamma x^{p-1} \circ y^{p-1} .
$$

We can now repeat the construction of the $z$ in (9) to obtain

$$
\begin{aligned}
& z D_{x}=y^{p-1} \circ m_{z}, \\
& z D_{y}=x^{p-1} \circ n_{z} .
\end{aligned}
$$

From these we can obtain (16) and (17). Hence we have concluded the proof of the theorem.

3. Let $\mathfrak{U}$ and $\mathfrak{U}^{*}$ be two simple nodal algebras that are equal as vector spaces and have the same + algebras. Let there be an even number of generators $x_{1}, \cdots, x_{2 r}$ with the multiplication in $\mathfrak{A}$ given by the $c_{i j}=x_{i} D\left(x_{j}\right)$ obtained in Theorem 1 and the multiplication in $\mathfrak{U}^{*}$ given by

$$
\begin{gathered}
c_{i i+r}^{\prime}=2, \\
c_{i j}^{\prime}=0
\end{gathered}
$$

for $i=1, \cdots, r$ and $j \neq i+r$. The algebra $\mathfrak{A}^{*}$ then falls into the class of simple 
nodal algebras defined by a skew-symmetric bilinear form and studied by Schafer [7].

Every derivation of $\mathfrak{A}$ must be a derivation of $\mathfrak{U}^{+}$. The derivations of $\mathfrak{U}^{+}$have been given by Jacobson $[2, \mathrm{p} .107]$ as

$$
f \rightarrow \sum_{1}^{2 r} \frac{\partial f}{\partial x_{k}} \circ a_{k}
$$

We shall denote this derivation by $\left(a_{1}, \cdots, a_{2 r}\right)$. Assume $\left(a_{1}, \cdots, a_{2 r}\right)$ is a derivation of $\mathfrak{A}$ and consider the possibility that $\left(b_{1}, \cdots, b_{2 r}\right)$ is a derivation of $\mathfrak{U}^{*}$ in which

$$
b_{i}=c_{i s}^{-1} \circ c_{i s}^{\prime} \circ a_{i}
$$

and $s$ is $i+r$ if $i \leqq r$ and is $i-r$ if $i>r$. In the same way we choose $t$ so $t=j+r$ or $j-r$ and $t \leqq 2 r$.

Consider the expression

$$
\sum_{k=1}^{2 r}\left(\frac{\partial c_{i j}^{\prime}}{\partial x_{k}} \circ b_{k}+\frac{\partial b_{i}}{\partial x_{k}} \circ c_{j k}^{\prime}+\frac{\partial b_{j}}{\partial x_{k}} \circ c_{k i}^{\prime}\right)
$$

obtained from Schafer's criteria [7, p. 312] that $\left(b_{1}, \cdots, b_{2 r}\right)$ be a derivative of $\mathfrak{I}^{*}$. We want to show that for all $i$ and $j(20)$ is 0 . By the choice of the $c_{i j}^{\prime}$ 's (20) can be reduced to

$$
\frac{\partial b_{i}}{\partial x_{t}} \circ c_{j t}^{\prime}+\frac{\partial b_{j}}{\partial x_{s}} \circ c_{s i}^{\prime}
$$

and by substituting the expressions (19) we have

$$
\begin{aligned}
& c_{j t}^{\prime} \circ c_{i s}^{\prime} \circ\left(\frac{\partial a_{i}}{\partial x_{t}} \circ c_{i s}^{-1}-\frac{\partial a_{j}}{\partial x_{s}} \circ c_{j t}^{-1}\right) \\
& +c_{j t}^{\prime} \circ c_{i s}^{\prime} \circ\left(\frac{\partial c_{i s}^{-1}}{\partial x_{t}} \circ a_{i}-\frac{\partial c_{j t}^{-1}}{\partial x_{s}} \circ a_{j}\right) .
\end{aligned}
$$

For our purposes we can drop the factor $c_{j t}^{\prime} \circ c_{i s}^{\prime}$, use the fact that if $q \in N$ then $\left(1+q^{p-1}\right)^{-1}=1-q^{p-1}$, and

$$
c_{i s} \circ c_{j t} \circ \frac{\partial c_{j t}}{\partial x_{s}}=c_{i s} \circ c_{j t} \circ \frac{\partial c_{i s}}{\partial x_{t}}=0
$$

to further reduce $(20)$ to

$$
\frac{\partial a_{i}}{\partial x_{t}} \circ c_{j t}-\frac{\partial a_{j}}{\partial x_{s}} \circ c_{i s}+\frac{\partial c_{j t}}{\partial x_{s}} \circ a_{j}-\frac{\partial c_{i s}}{\partial x_{t}} \circ a_{i}
$$

But the criteria that must be satisfied for $\left(a_{1}, \cdots, a_{2 r}\right)$ to be a derivation of $\mathfrak{U}$ is that $(21)$ be zero. Hence $\left(b_{1}, \cdots, b_{2 r}\right)$ is a derivation of $\mathfrak{U}^{*}$. From identities (14) of Schafer [7] we can now conclude that there is a $g$ such that 


$$
b_{i}=\left(\frac{\partial g}{\partial x_{s}}+\sigma_{i} \circ x_{s}^{p-1}\right) \circ c_{i s}^{\prime}
$$

in which $\sigma_{i}$ is in $\mathfrak{F}$. Therefore

$$
a_{i}=\left(\frac{\partial g}{\partial x_{s}}+\sigma_{i} \circ x_{s}^{p-1}\right) \circ c_{i s}
$$

Schafer has already proved [7, Theorem 8] that if the $a$ 's are defined as in (22) then they define a derivation.

We summarize as follows.

THEOREM 2. If $\mathfrak{A}$ is a simple, nodal, Lie-admissible noncommutative Jordan algebra of characteristic $p \neq 2$ such that $\mathfrak{A}^{+}$has an even number $n$ of generators then the derivation algebra $\mathfrak{D}(\mathfrak{U})$ of $\mathfrak{A}$ is the set of all mappings

$$
f \rightarrow \sum_{1}^{n} \frac{\partial f}{\partial x_{i}} \circ a_{i}
$$

in which the $a_{i}$ are defined as in (22). The dimension of $\mathfrak{D}(\mathfrak{U})$ is $p^{n}+n-1$.

We now investigate the algebras adj $\mathfrak{U}^{-},\left(\operatorname{adj} \mathfrak{U}^{-}\right)^{\prime}$ and $\left(\operatorname{adj} \mathfrak{A}^{-}\right)^{\prime \prime}$.

Using Schafer's result $\left[7\right.$, Theorem 7] we have $\mathfrak{U}^{-} / \mathfrak{F} 1 \cong \operatorname{adj} \mathfrak{U}^{-}$is of dimension $p^{2 r}-1$.

Since $D_{n} D_{m}-D_{m} D_{n}=D\left(n D_{m}\right)$ we can consider $\left(\operatorname{adj} \mathfrak{A}^{-}\right)^{\prime}$ as the set of all $D_{x}, x \in \mathfrak{A}^{-}$such that there are $y$ and $z$ in $\mathfrak{U}^{-}$with $x \equiv y D_{z}$ modulo $\mathfrak{F} 1$. Also $x_{i}^{2} D\left(x_{i+r}\right)=2 x_{i}$ implies $D\left(x_{i}\right) \in(\operatorname{adj} \mathfrak{U})^{\prime}$.

Before examining the dimension of $\left(\operatorname{adj} \mathfrak{A}^{-}\right)^{\prime}$ we consider a slightly more general situation.

Let $\mathfrak{I}$ be an ideal of $\mathfrak{A}^{-}$containing all of the generators $x_{1}, \cdots, x_{2 r}$. Let $m$ be a monomial of $\mathfrak{U}^{-}$that is not in $\mathfrak{F} 1$, and in which the exponent of $x_{1}$ is $i$ and $0 \leqq i<p-1$. Write $m=x_{1}^{i} \circ n$. Then

$$
\left(\frac{1}{i+1} x_{1}^{i+1} \circ n\right) D\left(x_{1+r}\right)=x_{1}^{i} \circ n \circ c_{11+r}
$$

If $i>0, c_{11+r} \in \mathfrak{F}$, or $x_{1+r}$ appears in $m$ with nonzero exponent then $x_{1}^{i} \circ n \circ c_{11+r}=x_{1}^{i} \circ n=m \in \mathfrak{I}$. Arguing on the arbitrariness of the choice of $x_{1}$ we see that all terms of degree greater than 0 are in $\mathfrak{I}$ except possibly those in which:

(1) every generator appears to either the 0 or $p-1$ power,

(2) $x_{i}$ has exponent $p-1$ if and only if $x_{i+r}$ has exponent $p-1$ for $i=1, \cdots, r$ and

(3) $x_{i}$ and $x_{i+r}$ have exponent $p-1$ if $c_{i i+r} \in \mathfrak{F}$.

However, assume such a term is $m$, and assume $x_{1}$ has exponent 0 in $m$ and $c_{11+r} \notin \mathfrak{F}$. Then from (23) we see that $m \equiv-\alpha_{1} m \circ x_{1}^{p-1} \circ x_{1+r}^{p-1}$ modulo $\mathfrak{~}$. 
This leaves us with at most two residue classes modulo $\mathfrak{I}$; the class containing 1 and the class containing $x_{i_{1}}^{p-1} \circ x_{i_{1}+r}^{p-1} \circ \cdots \circ x_{i_{t}}^{p-1} \circ x_{i_{t}+r}^{p-1}$ in which $\subseteq=\left\{i_{1}, \cdots, i_{t}\right\}$ is the set of all $i \leqq r$ such that $c_{i i+r} \in \mathfrak{F}$. If $\mathfrak{S}$ is empty then since

$$
x_{i} D\left(x_{i+r}\right)=1+\alpha_{i} x_{i}^{p-1} \circ x_{i+r}^{p-1}
$$

and $\alpha_{i} \neq 0$ there is at most one residue class, that one containing 1 .

We now let $\mathfrak{I}$ be the ideal in $\mathfrak{U}^{-}$such that $\mathfrak{I} \cong\left(\operatorname{adj} \mathfrak{U}^{-}\right)^{\prime}$. If $\mathfrak{S}=\varnothing$ by the above result we have $\mathfrak{I}=\mathfrak{A}^{-}$and $\left(\operatorname{adj} \mathfrak{A}^{-}\right)^{\prime}=\operatorname{adj} \mathfrak{A}$.

In case $\mathfrak{S} \neq \varnothing$ we first note that we have shown that $\mathfrak{I}$ contains all monomials and binomials of the form

$$
n \circ c_{i i+r},
$$

$i=1, \cdots, r$, and $n$ is a monomial without the factor $x_{i}^{p-1} \circ x_{i+r}^{p-1}$. To show that these are the only terms in $\mathfrak{I}$ we consider two monomials $n=x \circ x_{i}{ }^{u} \circ x_{i+r}^{v}$ and $m=y \circ x_{i}^{k} \circ x_{i+r}^{j}$ in which $x$ and $y$ are independent of $x_{i}$ and $x_{i+r}$. Every element of $\mathfrak{I}$ is a sum of terms of the form $n D_{m}$ and every $n D_{m}$ is a sum of terms of the form

$$
\begin{aligned}
& \left(x_{i}^{u} \circ x_{i+r}^{v}\right) D\left(x_{i}^{k} \circ x_{i+r}^{j}\right) \circ y \circ x \\
& \quad=y \circ x \circ x_{i}^{u+k-1} \circ x_{i+r}^{v+j-1} \circ(v k-u j) \circ c_{i i+r} .
\end{aligned}
$$

If $u+k-1=v+j-1=p-1$ then $v k-u j=0$. Hence every element of $\mathfrak{I}$ is a sum of terms of the form (24).

Now let $q$ be the product of all $x_{i}^{p-1}$ such that $i \in \mathfrak{S}$. If $q$ is in $\mathfrak{I}$ then it must be a sum of terms of the form (24). In fact we must have

$$
q=\sum q \circ n_{i} \circ c_{i i+r}
$$

in which $i \notin \mathfrak{S}, n_{i}$ is a polynomial independent of any of the generators in $q$. But this is a polynomial identity that holds in any scalar extension of $\mathfrak{F}$. Hence we can substitute field elements $\delta_{i}, \delta_{i+r}$ of some scalar extension $\mathcal{R}$ of $\mathfrak{F}$ for $x_{i}$ and $x_{i+r}, i \notin \mathfrak{S}$, so that $1+\alpha_{i} \delta_{i}^{p-1} \circ \delta_{i+r}^{p-1}=0$. But then the polynomial identity $q=0$ holds over $\mathfrak{R}$. Hence $q \notin \mathfrak{I}$.

We now show that $\left(\operatorname{adj} \mathfrak{U}^{-}\right)^{\prime}$ is simple. Let $\mathfrak{I}$ be an ideal of (adj $\left.\mathfrak{U}^{-}\right)^{\prime}$. To simplify the notation we will again actually work with an ideal in $\mathfrak{U}^{-}$and assume everything is reduced modulo $\mathfrak{F} 1$.

Let $\mathfrak{I}$ be the set of all polynomials in $\mathfrak{I}$ with a minimal number of terms in them. If the generator $x_{1}$ appears in any of these polynomials in $\mathfrak{I}$ choose one such polynomial $m$ in which $x_{1}$ appears to the minimal positive degree. Consider $m D\left(x_{1+r}^{2}\right)$ which is in $\mathfrak{I}$ and has fewer terms than $m$ unless $x_{1}$ appears with positive exponent in every term of $m$. Also, if any term is of degree greater than 1 in $x_{1}$ then we have a contradiction to our choice of $m$ to be of minimal degree in $x_{1}$. Hence we can assume $m=x_{1} \circ n$ in which $n$ is independent of $x_{1}$. By choosing $n$ 
to be of minimal positive degree in some second generator and avoiding the use of derivations $D_{y}$ for which $x_{1+r}$ appear in $y$ we can repeat the above argument finally obtaining a monomial $m$ in $\mathfrak{I}$ which is the product of distinct generators. If both $x_{i}$ and $x_{i+r}$ are in $m$ we can replace $m$ by $m D_{x_{i}}$. Hence we can assume in addition that the subscripts $i$ of the generator in $m$ satisfy $i \leqq r$. Write

$$
m=x_{i_{1}} \circ x_{i_{2}} \circ \cdots \circ x_{i_{t}}
$$

and apply successively the derivations

$$
D\left(x_{i_{1}+r}\right), D\left(x_{i_{2}+r} \circ c_{i_{1} i_{1}+r}^{-1}\right), \cdots, D\left(x_{i_{t}+r}^{2} \circ c_{i_{t-1} i_{t-1}+r}^{-1}\right)
$$

obtaining $x_{i_{t}+r} \in \mathfrak{I}$ and $x_{i_{+}+r} D\left(x_{i}^{2}\right)=2 x_{i} \in \mathfrak{I}$. Hence we can conclude that any generator that appears in a monomial of $\mathfrak{I}$ is in $\mathfrak{I}$. If $x_{i}$ is one such generator then for $i \neq j, x_{i} D\left(x_{i+r}^{2} \circ x_{j}\right)=2 x_{i+2} \circ x_{j}$ is in $\mathfrak{I}$ and $x_{j} \in \mathfrak{I}$. Therefore $\mathfrak{I}$ contains all generators. By the results above $\mathfrak{I}$ must be all of $\left(\operatorname{adj} \mathfrak{A}^{-}\right)^{\prime}$ and $\left(\operatorname{adj} \mathfrak{A}^{-}\right)^{\prime}$ is simple. We summarize in the following theorem.

TheOREM 3. If $\mathfrak{A}$ is a simple, Lie-admissible nodal noncommutative Jordan algebra of characteristic $p \neq 2$ with $2 r$ generators then (adj $\left.\mathfrak{U}^{-}\right)^{\prime}$ is a simple Lie algebra of dimension either $p^{2 r}-1$ or $p^{2 r}-2$ in the cases $\subseteq=\varnothing$ or $\subseteq \neq \varnothing$ respectively.

4. Let $\mathfrak{A}$ and $\mathfrak{U}^{*}$ be two nodal algebras that are equal as vector spaces and have the same + algebra. Let there be an odd number $n=2 r+1$, of generators $x_{1}, \cdots, x_{n}$ with the multiplication in $\mathfrak{A}$ given by $c_{i i+r}^{\prime}=2$ for $i=1, \cdots, r$ and all other $c_{i j}=0$.

Let $\left(a_{1}, \cdots, a_{n}\right)$ be a derivation of $\mathfrak{A}$. Just as in the previous section we can show $\left(b_{1}, \cdots, b_{2 r-2}, 0,0,0\right)$ is a derivation of $\mathfrak{A}^{*}$ if

$$
b_{i}=c_{i s}^{-1} \circ c_{i s}^{\prime} \circ a_{i}
$$

for $i=1, \cdots, r-2$. Therefore we must have

$$
a_{i}=\left(\frac{\partial g}{\partial x_{s}}+\sigma_{i} \circ x_{s}^{p-1}\right) \circ c_{i s}
$$

for $i=1, \cdots, r-1$. Here though, $\sigma_{i}$ can apparently be any polynomial in $\mathcal{F}\left[x_{2 r-1}, x_{2 r}, x_{2 r+1}\right]$. To obtain further restrictions on the $\sigma_{i}$ we examine derivations of the form

$$
\left(\sigma_{1} \circ x_{1+r}^{p-1} \circ c_{11+r}, \cdots, \sigma_{2 r-2} \circ x_{r-1}^{p-1} \circ c_{2 r-2 r-2}, a_{2 r-1}, a_{2 r}, a_{2 r+1}\right) .
$$

We now use identity (5) of Schafer [7] with $i \leqq r-2$ and $j \geqq 2 r-1$ to obtain

$$
\sum_{2 r-1}^{n} \frac{\partial \sigma_{i}}{\partial x_{k}} \circ x_{i+r}^{p-1} \circ c_{j k}+\frac{\partial a_{j}}{\partial x_{i+r}} \circ c_{i+r i}=0 .
$$

Since $c_{i+r}$ is nonsingular and $\partial a_{j} / \partial x_{i+r}$ is of degree at most $p-2$ in $x_{i+r}$ we 
must have $\partial a_{j} / \partial x_{i+r}=0$ and $a_{j}$ independent of $x_{i+r}$. Interchanging $i$ and $i+r$ in (26) we see $a_{j}$ is also independent of $x_{i}$. Hence $a_{j}$ is a polynomial in $\mathfrak{F}\left[x_{2 r-1}, x_{2 r}, x_{2 r+1}\right]$.

We now select $j$ in (26) to be $2 r$. Then

$$
\frac{\partial \sigma_{i}}{\partial x_{2 r-1}} x_{i+r}^{p-1} c_{2 r 2 r-1}+\frac{\partial \sigma_{i}}{\partial x_{2 r+1}} \circ x_{i+r}^{p-1} \circ c_{2 r 2 r+1}=0 .
$$

Since $x_{2 r}^{p-1}$ is a factor of $c_{2 r 2 r+1}$ and $\sigma_{i}$ is independent of $x_{i+r}$ we must have

$$
\begin{aligned}
\frac{\partial \sigma_{i}}{\partial x_{2 r+1}} \circ c_{2 r 2 r+1} & =0 \\
\frac{\partial \sigma_{i}}{\partial x_{2 r-1}} & =0 .
\end{aligned}
$$

Hence $\sigma_{i}$ is independent of $x_{2 r-1}$. In the same way we see that $\sigma_{i}$ is independent of $x_{2 r}$. Now by the first relationship in (27) we have $\sigma_{i}$ independent of $x_{2 r+1}$ and $\sigma_{i} \in \mathfrak{F}$.

We can now confine our attention to finding the derivations of an algebra $\mathfrak{A}$ with three generators $x, y, z$ in which multiplication is defined by

$$
\begin{aligned}
& y D_{x}=1+\gamma x^{p-1} \circ y^{p-1}=d_{12}, \\
& z D_{x}=y^{p-1} \circ\left(1+\beta z^{p-1}\right)=d_{13}, \\
& z D_{y}=\alpha x^{p-1} \circ\left(1+\beta z^{p-1}\right)=d_{23} .
\end{aligned}
$$

Let $\left(a, a_{2}, a_{3}\right)$ be a derivation of $\mathfrak{A}$. Since there are derivations of the form

$$
b_{i}=\frac{\partial g}{\partial x} \circ d_{i 1}+\frac{\partial g}{\partial y} \circ d_{i 2}+\frac{\partial g}{\partial z} \circ d_{i 3}
$$

[7, Theorem 8] and $a_{1} \circ d_{12}^{-1}=\partial g / \partial y$ can be solved to within a multiple 0 $y^{p-1}[7$, Lemma 1], we can subtract off the derivation induced by $g$ and assume $a_{1}=\delta \circ y^{p-1}$ in which $\delta$ is a polynomial in $x$ and $z$. Using the same lemma we can solve $-\mu^{-1} \circ \delta=\partial g / \partial z$ to within a multiple of $z^{p-1}$ and such that $g$ is in $\mathscr{F}[x, z]$. Subtracting off the derivation corresponding to this $y$ leaves us with $a_{1}=\delta_{0} \circ z^{p-1} \circ y^{p-1}$ in which $\delta_{0}$ is a polynomial in $x$.

The three conditions [7] that $\left(a_{1}, a_{2}, a_{3}\right)$ be a derivation can be written in the form

$$
\begin{aligned}
& -\frac{\partial\left(d_{12}^{-1} a_{2}\right)}{\partial y} \circ d_{12}^{2}+\frac{\partial a_{1}}{\partial x} \circ d_{21}+\frac{\partial a_{2}}{\partial z} \circ d_{31}+\frac{\partial a_{1}}{\partial z} \circ d_{23}=0 \\
& -y^{p-1} \circ \frac{\partial\left(\mu^{-1} a_{3}\right)}{\partial z} \circ \mu^{2}+\frac{\partial d_{13}}{\partial y} \circ a_{2}+\frac{\partial a_{1}}{\partial y} \circ d_{32}+\frac{\partial a_{3}}{\partial y} \circ d_{21}=0
\end{aligned}
$$




$$
\begin{aligned}
-\alpha x^{p-1} \circ \frac{\partial\left(\mu^{-1} a_{3}\right)}{\partial z} \circ \mu^{2}+\frac{\partial d_{23}}{\partial x} \circ a_{1} & +\frac{\partial a_{2}}{\partial y} \circ d_{32} \\
& +\frac{\partial a_{2}}{\partial x} \circ d_{31}+\frac{\partial a_{3}}{\partial x} \circ d_{12}=0
\end{aligned}
$$

in which $\mu=1+\beta z^{p-1}$.

The last three terms of (28) are in $y^{p-1} \circ \mathfrak{A}$ since $a_{1}$, and $d_{31}$ are. Hence both $-d_{12}^{2} \circ \partial\left(d_{12}^{-1} \circ a_{2}\right) / \partial y$ and $\partial\left(d_{12}^{-1} \circ a_{2}\right) / \partial y$ are in $y^{p-1} \circ \mathfrak{A}$. But the second polynomial is of degree at most $p-2$ in $y$ and hence is 0 . Therefore there is a polynomial $\delta_{1}$ independent of $y$ and s:ich that $a_{2}=\delta_{1} \circ d_{12}$.

Identity (28) now reduces to

$$
\begin{aligned}
\mu^{-1} \circ & \frac{\partial \delta_{0}}{\partial x} \circ y^{p-1} \circ z^{p-1}+\frac{\partial \delta_{1}}{\partial z} \circ y^{p-1} \\
& -\alpha y^{p-1} \circ x^{p-1} \circ \frac{\partial\left(\delta_{0} z^{p-1}\right)}{\partial z}=0 .
\end{aligned}
$$

Arguing on the degree of $z$ in each term of (31) we can conclude $\partial \delta_{0} / \partial x=0$ and $\delta_{0}$ is independent of $x$. But $\delta z^{p-1} \circ y^{p-1}=\delta z^{p-1} \circ d_{13}$ and

$$
\left(\delta z^{p-1} \circ d_{13}, \delta z^{p-1} \circ d_{23}, 0\right)
$$

is a derivation of $A$. Subtracting off this derivation we can assume $a_{1}=\delta_{0}=0$.

From(31), since $\delta_{1}$ is independent of $y$, we also get $\delta_{1}$ independent of $z$, i.e., $\delta_{1}$ is a polynomial in $\mathfrak{F}[x]$. Therefore we can find a polynomial $g$ in $\mathfrak{F}[x]$ that is a solution of $\delta_{1} \circ d_{12}=d_{21} \circ \partial g / \partial x$ to within a constant multiple of $x^{p-1}$, say $\eta x^{p-1}$.

Subtracting off the derivation

$$
\left(0, d_{21} \circ \frac{\partial g}{\partial x}-\eta x^{p-1} \circ d_{21}, d_{31} \circ \frac{\partial g}{\partial x}-\eta x^{p-1} \circ d_{31}\right)
$$

we can assume $a_{1}=a_{2}=0$. Equations (29) and (30) now reduce to

$$
\begin{aligned}
& -y^{p-1} \circ \frac{\partial\left(\mu^{-1} \circ a_{3}\right)}{\partial z} \circ \mu^{2}+\frac{\partial a_{3}}{\partial y} \circ d_{21}=0, \\
& -\alpha x^{p-1} \circ \frac{\partial\left(\mu^{-1} \circ a_{3}\right)}{\partial z} \circ \mu^{2}+\frac{\partial a_{3}}{\partial x} \circ d_{12}=0 .
\end{aligned}
$$

Since $d_{21}$ is nonsingular we can argue on the degree of $y$ to get $\partial a_{3} / \partial y=0$ and $a_{3}$ is independent of $y$. In the same manner $a_{3}$ is independent of $x$. But then $\mu^{-1} a_{3}$ is independent of $z$. Hence $a_{3}=\eta \mu$ for $\eta \in \mathfrak{F}$.

By direct substitution in (32) it can be seen that $(0,0, \eta \mu)$ is a derivation of $\mathfrak{A}$. We investigate to see if it is of the form $\left(a_{1}, a_{2}, a_{3}\right)$ in which 


$$
\begin{aligned}
& a_{1}=\left(\frac{\partial g}{\partial y}+\alpha_{2} y^{p-1}\right) \circ d_{12}+\left(\frac{\partial g}{\partial z}+\alpha_{3} z^{p-1}\right) \circ d_{13} \\
& a_{2}=\left(\frac{\partial g}{\partial x}+\alpha x^{p-1}\right) \circ d_{21}+\left(\frac{\partial g}{\partial z}+\alpha_{3} z^{p-1}\right) \circ d_{23} \\
& a_{3}=\left(\frac{\partial g}{\partial x}+\alpha_{1} x^{p-1}\right) \circ d_{31}+\left(\frac{\partial g}{\partial y}+\alpha_{2} y^{p-1}\right) \circ d_{32}
\end{aligned}
$$

If $a_{1}=a_{2}=0$ then $a_{1} \equiv 0$ modulo $y^{p-1}$ and $\partial g / \partial y \in y^{p-1} \circ \mathfrak{A}$. Hence $g$ is independent of $y$. In the same way $g$ is independent of $x$. Therefore $a_{3}=-x^{p-1} \circ y^{p-1} \circ \mu \circ\left(\alpha_{1}+\alpha \alpha_{2}\right)$ which is not of the form $\eta \mu$ for $\eta \in \mathfrak{F}$.

We can now conclude:

THEOREM 4. Let $\mathfrak{A}$ be a simple, nodal, Lie-admissible noncommutative Jordan algebra of characteristic $p \neq 2$ with $2 r+1$ generators; then the derivation algebra $\mathfrak{D}(\mathfrak{U})$ of $\mathfrak{U}$ is the set of all mappings

$$
f \rightarrow \sum_{1}^{n} \frac{\partial f}{\partial x_{i}} \circ a_{i}
$$

in which

$$
\begin{aligned}
a_{i} & =\sum_{j=1}^{n}\left(\frac{\partial g}{\partial x_{j}}+\alpha_{j} x_{j}^{p-1}\right) \circ c_{i j} \\
a_{2 r+1} & =\sum_{2 r-1}^{2 N}\left(\frac{\partial g}{\partial x_{i}}+\alpha_{i} x_{i}^{p-1}\right) \circ c_{2 r+1 i}+\eta \mu
\end{aligned}
$$

for $i=1, \cdots, 2 r$. (In case $i<2 r-1$ then $a_{i}$ reduces to a single summand.) The dimension of $\mathfrak{D}(\mathfrak{Q})$ is $p^{2 r+1}+2 r+1$.

To determine the dimension of $\left(\operatorname{adj} \mathfrak{A}^{-}\right)^{\prime}$ we proceed as in the even-dimensional case. Let $\mathfrak{I}$ be an ideal of $\mathfrak{A}$ - containing all of the generators $x_{1}, \cdots, x_{2 r}$. Using only the generators $x_{1}, \cdots, x_{2 r-2}$ we have the result from the even-dimensional case that the only possible residue classes modulo $\mathfrak{I}$ are the classes determined by 1 and the polynomials of the form $q m$ in which $q=x_{1}^{p-1} \circ \cdots \circ x_{2 r-2}^{p-1}$ and $m$ is a polynomial in $x_{2 r-1}, x_{2 r}$ and $x_{2 r+1}$. We adopt the notation above using $x, y$ and $z$, $x_{2 r-1}, x_{2 r}$, and $x_{2 r+1}$ respectively. Assume $m$ is a monomial and $m=x^{i} \circ n, n$ independent of $x$ and $i<p-1$; then

$$
\left(\frac{1}{i+1} q \circ x^{i+1} \circ n\right) D_{y}=-q \circ m \circ d_{12} .
$$

Also if $m=y^{i} \circ n, n$ independent of $y$ and $i<p-1$ then

$$
\left(\frac{1}{i+1} q \circ y^{i+1} \circ n\right) D_{x}=q \circ m \circ d_{12} \text {. }
$$


Hence the only remaining residue classes of $\mathfrak{I}$ to examine are those determined by $q \circ x^{p-1} \circ y^{p-1} \circ n$ in which $n$ is a polynomial in $z$. However the equation

$$
\begin{aligned}
\left(q \circ x^{p-1} \circ t\right) D_{x} & =q \circ x^{p-1} \circ y^{p-1} \circ \frac{\partial t}{\partial y} \circ \mu \\
& =q \circ x^{p-1} \circ y^{p-1} \circ n
\end{aligned}
$$

can be solved for $t$, a polynomial in $\mathfrak{\&}[z]$, to within a scalar multiple of $q \circ x^{p-1} \circ y^{p-1} \circ z^{p-1}$. Hence the only possible residue class of $\Im$ is that containing $q \circ x^{p-1} \circ y^{p-1} \circ z^{p-1}$. If $\mathfrak{S}=\varnothing$ (the set of all $i=1, \cdots, r$ such that $c_{i i+r} \in \mathfrak{F}$ ) and $\beta \neq 0$ then as we have seen in the even-dimensional case $q \circ x^{p-1} \circ y^{p-1} \in \mathfrak{I}$ and $\left(q \circ x^{p-1} \circ z\right) D_{x}=\left(q \circ x^{p-1} \circ y^{p-1} \circ \mu\right) \in \mathfrak{I}$. Therefore $q \circ x^{p-1} \circ y^{p-1} \circ z^{p-1} \in \mathfrak{I}$.

If $\mathfrak{I}$ is the ideal in $\mathfrak{A}^{-}$such that $\mathfrak{A}^{-} / \mathfrak{F} 1$ is isomorphic to (adj $\left.\mathfrak{A}^{-}\right)^{\prime}$ then we can show, exactly as in the even-dimensional case, that $q \circ x^{p-1} \circ y^{p-1} \circ z^{p-1}$ is not in $\mathfrak{I}$ if either $\mathfrak{S} \neq \varnothing$ or $\beta=0$. Hence (adj $\left.\mathfrak{A}^{-}\right)^{\prime}$ is of dimension $p^{2 r-1}-1$ or $p^{2 r+1}-2$.

We now examine the ideals of $\left(\operatorname{adj} \mathfrak{A}^{-}\right)^{\prime}$. Let $\mathfrak{I}$ be an ideal of $\left(\operatorname{adj} \mathfrak{A}^{-}\right)^{\prime}$. (We again use the notation of $\mathfrak{A}^{-}$.) As in the even-dimensional case we can assume there are polynomials of the form $x_{i} \circ m$ for any $i \leqq 2 r-1$ and in which $m$ is a polynomial in $\mathfrak{F}[x, y, z]$.

Consider those polynomials $x \circ m$. If $m$ is in $\mathfrak{F}[x]$ we choose a $k$ so that

$$
\left(x_{1} \circ m\right) D\left(x_{1} \circ x_{1+r} \circ x^{k}\right)=x_{1} \circ x^{p-1} \text {. }
$$

If $m \notin \mathfrak{F}[x]$, write

$$
m=m_{1}+\sum_{k}^{p-1} x^{i} \circ n_{i}
$$

in which $m_{1}$ is a polynomial in $x$, every term of every nonzero $n_{i}$ has either a $y$ or $z$ in it and some $n_{i} \neq 0$. If $k \neq 0$ then

$$
\left(x_{1} \circ m\right) D\left(x^{p-k}\right)=-k x^{p-1} \circ n_{k} D_{x} \circ x_{1} \neq 0
$$

is in $\mathfrak{I}$. If $k=0$ then

$$
\left(x_{1} \circ m\right) D\left(x^{p-1}\right)=\left(-n_{0} D_{x} \circ x^{p-2}-x^{p-1} \circ n_{1} D_{x}\right) \circ x_{1} \neq 0
$$

is in $\mathfrak{I}$. If $n_{0} D_{x}$ and $n_{1} D_{x}$ are in $\mathfrak{F}$ then as above we can conclude $x_{1} \circ x^{p-1} \in \mathfrak{I}$. If $n_{0} D_{x}$ is in $\mathfrak{F}$ but $n_{1} D_{x}$ is not then

$$
\left(x_{1} \circ m\right) D\left(x^{p-1}\right) D_{x}=-x^{p-1} \circ x_{1} \circ n_{1} D_{x} D_{x} \neq 0
$$

is in $\mathfrak{I}$. If $n_{0} D_{x} \notin \mathfrak{F}$ then

$$
\left(x_{1} \circ m\right) D\left(x^{p-1}\right) D\left(x^{2}\right)=-2 n_{0} D_{x}^{2} \circ x^{p-1} \circ x_{1} \neq 0
$$

is in $\mathfrak{I}$. In any case, there is a polynomial $x_{1} \circ x^{p-1} \circ m$ in $\mathfrak{I}$ in which $m \in \mathfrak{F}[y, z]$. If $m$ is in $\mathfrak{F}$ we can proceed as in the even-dimensional case to show that $x_{1}, \cdots, x_{2 r}$ are in $\mathfrak{I}$. 
If $m$ is independent of $y$ then assume $m$ is such a polynomial of minimal degree in $z$. We have

$$
\left(x_{1} \circ x^{p-1} \circ m\right) D_{x}^{p}=x_{1} \circ x^{p-1} \circ \frac{\partial m}{\partial z}
$$

By the minimality of the degree of $z$ in $m$ we have $\partial m / \partial z=0, m \in \mathfrak{F}$ and $x_{1} \circ x^{p-1} \in \mathfrak{I}$.

If $m$ is not independent of $y$ then

$$
\left(x_{1} \circ x^{p-1} \circ m\right) D_{y}^{p-2}=x_{1} \circ x \circ m
$$

is in $\mathfrak{I}$. Let $k$ be the smallest exponent of $y$ in $m$. If $k=0$ then $\left(x_{1} \circ x \circ m\right) D_{z}$ $=\alpha x_{1} \circ y^{p-1} \circ m \circ \mu=x_{1} \circ y^{p-1} \circ n$ is in $\mathfrak{J}$ for some polynomial $n$ in $\mathfrak{F}[z]$. If $k \neq 0$ then $\left(x_{1} \circ x \circ m\right) D\left(y^{p-k}\right)=k x_{1} \circ y^{p-1} \circ n$ is in $\mathfrak{I}$ for some polynomial $n$ in $\mathfrak{F}[z]$. Choose $n$ to be of minimal degree in $z$. Then as above we can show $n$ is in $\mathfrak{F}$ and $x_{1} \circ y^{p-1} \in \mathfrak{I}$.

Thus either $x_{1} \circ x^{p-1}$ or $x_{1} \circ y^{p-1}$ is in $\mathfrak{J}$. As in the even-dimensional case this implies $x_{1}, \cdots, x_{2 r}$ are in $\mathfrak{I}$. Hence from our conclusion above on such ideals $\mathfrak{I}$ we have (adj $\left.\mathfrak{A}^{-}\right)^{\prime}$ is simple. Thus

THeOREM 5. If $\mathfrak{U}$ is a simple, Lie-admissible nodal noncommutative Jordan algebra of characteristic $p \neq 2$ with $2 r+1$ generators then (adj $\left.\mathfrak{U}^{-}\right)^{\prime}$ is a simple Lie-algebra. The dimension of (adj $\left.\mathfrak{A}^{-}\right)^{\prime}$ is $p^{2 r-1}-1$ if $\mathfrak{S}=\varnothing$ and $\beta \neq 0$ and is $p^{2 r+1}-2$ if either $\mathfrak{S} \neq 0$ or $\beta=0$.

\section{REFERENCES}

1. Richard Block, New simple Lie algebras of prime characteristic, Trans. Amer. Math. Soc. 89 (1958), 421-449.

2. Nathan Jacobson, Classes of restricted Lie algebras of characteristic p. II, Duke Math. J. 10 (1943), 107-121.

3. L. A. Kokoris, Some nodal noncommutative Jordan algebras, Proc. Amer. Math. Soc. 9 (1958), 164-166.

4. - Simple nodal noncommutative Jordan algebras, Proc. Amer. Math. Soc. 9 (1958), 652-654.

5. - Nodal noncomutative Jordan algebras, Canad. J. Math. 12 (1960), 488-492.

6. R. D. Schafer, On noncommutative Jordan algebras, Proc. Amer. Math. Soc. 9 (1958), 110-117.

7. - Nodal noncommutative Jordan algebras and simple Lie algebras of characteristic $p$, Trans. Amer. Math. Soc. 94 (1960), 310-326.

INSTITUTE FOR Defense ANALyses,

Princeton, New Jersey

Michigan State University,

East Lansing, Michigan 